\title{
Long-Term Care Facility
}

National Cancer Institute

\section{Source}

National Cancer Institute. Long-Term Care Facility. NCI Thesaurus. Code C53530.

A healthcare facility that provides rehabilitative, restorative, and/or ongoing skilled

nursing care to patients or residents in need of assistance with activities of daily living. 